Check for updates

Cite this: RSC Adv., 2017, 7, 33510

\title{
Vertically aligned PANI nanorod arrays grown on graphene oxide nanosheets for a high-performance $\mathrm{NH}_{3}$ gas sensor
}

\author{
Jinwei Wei, $\xi^{a}$ Bo Liang, $\stackrel{\ddagger}{+}^{a}$ Qingpeng Cao, ${ }^{a}$ Chentian Mo, ${ }^{a}$ Youming Zheng ${ }^{b}$ \\ and Xuesong Ye (iD *ac
}

In this paper, vertically aligned PANI nanorod arrays were successfully prepared on GO nanosheets at low temperature via a dilute polymerization method, in which the GO nanosheets with abundant hydrophilic groups were employed to support the heterogeneous growth of uniformly-distributed PANI nanorod arrays. The acquired nanocomposite was characterized by scanning electron microscopy (SEM), transmission electron microscopy (TEM), Fourier transform infrared spectroscopy (FTIR), Raman spectroscopy, and X-ray photoelectron spectroscopy (XPS). $\mathrm{NH}_{3}$ sensing performances of the as-prepared nanocomposite were carefully examined. With the help of the enhanced synergistic effect derived from the large number of $p-n$ heterojunctions in the deliberately-designed microstructure, the device exhibited an enhanced response to $\mathrm{NH}_{3}$ vapor (14.63 at $50 \mathrm{ppm}$ ), wide dynamic range (1-6400 ppm), and fast response and recovery processes, in comparison to pure PANI nanofibers. The impact of the drop-coating amount of the sensing nanocomposite on the sensing performances was also investigated to find out the suitable amount for fabricating a high-performance ammonia sensor. Herein, the superior performances of the proposed wellorganized microstructure make it an effective nanocomposite for gas sensing applications.

Received 25th April 2017

Accepted 7th June 2017

DOI: $10.1039 / \mathrm{c} 7 \mathrm{ra0} 4636 \mathrm{~g}$

rsc.li/rsc-advances
At present, more efforts have been made to decorate graphene or GO with functional nanomaterials for higher sensitivity and better selectivity in gas sensors. The decoration of CVD grown graphene with gold nanoparticles using the galvanic displacement method has been proposed for $\mathrm{H}_{2} \mathrm{~S}$ monitoring. ${ }^{7}$ In addition, metal oxide nanomaterials were combined with graphene for gas sensor fabrication with excellent gas sensing properties. A high performance $\mathrm{NO}_{2}$ gas sensor has been successfully fabricated using $\mathrm{ZnO}$ and $\mathrm{Au}$ nanoparticles modified reduced graphene oxide ternary hybrids, showing high sensitivity, fast response and recovery rate. ${ }^{8}$ Another $\mathrm{NO}_{2}$ gas sensor with a high sensitivity and low detection of level has been achieved by using self-assembled graphene/polymer composite nanofibers as sensitive material. ${ }^{9}$

Among all the functional nanomaterials exploited for sensitivity enhancement, conducting polymers such as polyaniline (PANI), polypyrrole and polythiophene presented excellent synergistic effect when introduced into graphene or RGO based gas sensors. ${ }^{10,11}$ There have been a number of scientific reports on graphene/PANI nanocomposite used in gas sensor fabrication, which is largely attributed to the facile synthesis and environmental stability of PANI. Graphene/PANI nanocomposite synthesized by chemical oxidation polymerization was used for $\mathrm{NH}_{3}$ determination, showing a wide linear range of concentrations from 1 to $6400 \mathrm{ppm} \cdot{ }^{12}$ Besides, PANI with fibrous morphology in the graphene/PANI nano-composite has been achieved to develop a novel $\mathrm{H}_{2}$ sensor. ${ }^{13}$ Despite some 
improvements of sensing performances, the inherent agglomeration of graphene in chemical synthesis processes, due to high surface energy, was very difficult to overcome if not impossible. Therefore, few well-organized microstructures of graphene/PANI nanocomposites have been achieved yet.

Although various graphene/PANI or RGO/PANI nanocomposites were successfully exploited in gas sensor fabrications ${ }^{\mathbf{1 2 , 1 3}}$ and supercapacitor electrodes, ${ }^{2,3,14}$ little attention, to the best of our knowledge, has been paid to the facile chemical synthesis of well-organized GO/PANI nano-composite and its gas sensing performances. Here, we presented a controllable in situ polymerization method for vertically aligned PANI nanorod arrays (PNrA) grown on GO nanosheets (PNrA/GO) at low temperature. ${ }^{15,16}$ It suggested that GO nanosheets with abundant hydroxyl and carboxyl groups could provide active nucleation sites for PANI growth, leading to a relatively uniform distribution in PANI nanorod arrays. Therefore, the asfabricated ammonia sensor, with the proposed sensitive nanocomposite and a well-organized micro-structure design, exhibited greater overall performances, such as higher sensitivity and wider detection range, than those of PANI/RGO based gas sensors reported previously. ${ }^{11,17,18}$

\section{Experimental section}

\section{Characterizations}

For morphologic characterization, both the prepared nanocomposites and gas sensor architectures were observed by field-emission scanning electron microscopy (FESEM, Hitachi SU8010) with the accelerating voltage ranging from 0.1 to $30 \mathrm{kV}$. Transmission electron microscopy (TEM) characterization was made on a JEOL JEM-1200EX electron microscopy with an accelerating voltage of $200 \mathrm{kV}$. For chemical composition analysis, Fourier transform infrared spectra (FT-IR) of GO, PANI nanofibers, and the synthesized PNrA/GO were recorded by Tensor 27 from Bruker with the wavenumber range of 7800 $\mathrm{cm}^{-1}$ to $400 \mathrm{~cm}^{-1}$. Raman spectra were obtained using a LabRAM HR Evolution system with a $532 \mathrm{~nm}$ wavelength laser, which was bought from Horiba Jobin Yvon. The XPS spectra of the products were detected by VG ESCALAB MARK II spectrometer with the energy resolution to be $0.8 \mathrm{eV}$.

\section{Materials}

Graphene oxide (GO) powder was purchased from Nanjing XFNANO Materials Tech Co., Ltd. (Nanjing, China). Ammonium persulfate (APS) was obtained from Aladdin Ltd. (Shanghai, China). All other reagents were purchased from Sinopharm Chemical Reagent Co., Ltd. (Shanghai, China) and were reagent grade. Deionized (18 $\mathrm{M} \Omega \mathrm{cm}^{-1}$ ) water was used for all aqueous solutions.

\section{Preparation of vertically aligned PANI nanorod arrays on GO nanosheets}

The successful preparation was accomplished by in situ dilute polymerization of aniline for vertically aligned PANI nanorod arrays uniformly-distributed on graphene oxide nanosheets (abbreviated as PNrA/GO).

In detail, our approach for PNrA/GO can be presented as follows: $21.6 \mathrm{mg}$ of $\mathrm{GO}$ was dispersed in $15 \mathrm{~mL}$ of deionized water with the assistance of powerful ultrasonication for $0.5 \mathrm{~h}$, obtaining a brown solution. Then $83.82 \mathrm{mg}$ of aniline was mixed with $22.5 \mathrm{~mL}$ of $\mathrm{HClO}_{4}(1.0 \mathrm{M})$, followed by magnetic stirring for $0.5 \mathrm{~h}$ at room temperature. And $136.88 \mathrm{mg}$ of APS was dispersed in $22.5 \mathrm{~mL}$ of $\mathrm{HClO}_{4}(1.0 \mathrm{M})$ with continuous stirring, followed by cold storage for $0.5 \mathrm{~h}$ at $0{ }^{\circ} \mathrm{C}$. Afterwards, the obtained GO dispersed solution was rapidly added to the aniline mixture and the resulting mixture was treated with magnetic stirring for another $0.5 \mathrm{~h}$ at $0{ }^{\circ} \mathrm{C}$. The APS solution was then rapidly mixed with the above GO/aniline mixture and the resulting suspension with an aniline concentration of $0.015 \mathrm{M}$, a ratio of [aniline]/[GO] to be $3.9: 1$ and a ratio of [aniline]/[APS] to be $1.5: 1$ was treated by magnetic stirring for $24 \mathrm{~h}$ at $0{ }^{\circ} \mathrm{C}$. For comparison, the $\mathrm{PNrA} / \mathrm{GO}$ were also successfully prepared in suspensions with a ratio of [aniline]/ [GO] to be $5.2: 1,2.6: 1$ and $1.3: 1$, in which the aniline concentration was $0.02 \mathrm{M}, 0.01 \mathrm{M}$ and $0.005 \mathrm{M}$, respectively and the ratio of [aniline]/[APS] remained unchanged. Thus, the obtained PNrA/GO nano-composites were denoted as PNrA/GO (5.2:1), PNrA/GO (3.9:1), PNrA/GO (2.6:1) and PNrA/GO (1.3:1), respectively. After the polymerization process, centrifugal purification was employed to remove the remaining reactants and other by-products. For further comparison, the pure PANI nanofibers and PANI nanofibers/GO nanocomposite were polymerized in $0.1 \mathrm{M}$ aniline solution in the absence and presence of GO nanosheets by the same approach as mentioned above, and thus the prepared products were respectively named as $\mathrm{PNF}$ and $\mathrm{PNF} / \mathrm{GO}$ for short.

To illustrate the growth process of the nanocomposite, the PNrA/GO synthesized in suspensions with the aniline concentration of $0.015 \mathrm{M}$, a ratio of [aniline]/[GO] to be $3.9: 1$ and a ratio of [aniline]/[APS] to be $1.5: 1$ was prepared at the reaction time of $1 \mathrm{~h}, 4 \mathrm{~h}, 8 \mathrm{~h}$ and $24 \mathrm{~h}$.

\section{Fabrication of gas sensor and the evaluation of sensing properties}

After purification, PNrA/GO achieved good stability and uniform dispersion in aqueous state at room temperature to greatly facilitate the sensor fabrication processes. Then, the asprepared ammonia gas sensor was obtained by drop-coating the PNrA/GO onto the interdigitated electrodes, followed by thermal treatment in vacuum oven at $45{ }^{\circ} \mathrm{C}$ for $8 \mathrm{~h}$. The interdigitated electrodes was fabricated on $\mathrm{Si} / \mathrm{SiO}_{2}$ substrate with the size of $4.3 \mathrm{~mm} \times 4.0 \mathrm{~mm} \times 1.0 \mathrm{~mm}$, and both the width and gaps between the gold lines were $3 \mu \mathrm{m}$. In comparison, the ammonia sensor based on pure PANI nanofibers, GO nanosheets and PANI nanofibers/GO composite were fabricated using the same method as mentioned above, respectively.

Then, gas sensing evaluations were conducted by recording the relative real-time resistance changes when the gas sensor were exposed to different concentrations of $\mathrm{NH}_{3}$ at room temperature. The typical testing procedures can be conducted 
as follows: firstly, the gas sensor was kept still in the air till the resistance response reached its steady state, where the resistance was recorded as $R_{\text {air }}$; then, the sensor was immediately opposed to ammonia diluted in a specific concentration using $\mathrm{N}_{2}$ as carrier gas, and we assigned the resistance here as $R_{\text {gas }}$; thirdly, it was withdrawn out to the surrounding air after that the $R_{\text {gas }}$ got its equilibrium state. The standard $\mathrm{NH}_{3}$ (50 $000 \mathrm{ppm}, \mathrm{N}_{2}$ as carrier gas) and pure $\mathrm{N}_{2}$ were purchased from MINXINGQITI (Hangzhou, China) and different concentrations of $\mathrm{NH}_{3}$ can be easily and accurately obtained by using Sabio Gas Dilution Calibrator (Model 4010L, USA). Additionally, the resistance of the as-fabricated interdigitated electrodes were recorded and stored using digital multimeter (Agilent 34410A, USA), which was connected to PC by USB to RS232 serial port cable.

It is noted that the sensor response of ammonia sensor is defined as $S=\left(\left(R_{\text {gas }}-R_{\text {air }}\right) / R_{\text {air }}\right)$, in which the $R_{\text {gas }}$ represents the resistance in target gas and the $R_{\text {air }}$ for that in air as mentioned above. Moreover, the response time is defined as the consumed time at which the magnitude reaches $90 \%$ of the total response signal and the recovery time denotes the time required for a sensor to restore $90 \%$ of the total resistance change.

\section{Results and discussion}

\section{Characterization of vertically aligned PANI nanorod arrays on graphene oxide}

Here, a low-temperature dilute polymerization method was utilized to grow vertically aligned PANI nanorod arrays on GO nanosheets and the acquired nanocomposite was drop-coated onto the interdigitated electrodes for gas sensor fabrication. Scanning electron microscopy (SEM) images were collected to characterize the surface morphology of PNrA/GO and pure PANI nanofibers, respectively. Fig. $1 \mathrm{a}$ and $\mathrm{b}$ revealed that the dilutepolymerized vertically aligned PANI nanorod arrays with the crystal height to be several tens of nanometers were uniformly distributed on the graphene oxide nanosheets. In comparison, Fig. 1c and $d$ revealed that pure PANI nanofibers and PANI nanofibers/GO composite were successfully synthesized with the removal and addition of GO nanosheets and that the homogeneous polymerization of aniline monomers dominated the main process when the aniline concentration was raised up to $0.1 \mathrm{M}$, leading to randomly interconnected PANI nanofibers.

Moreover, it was observed that electrode gaps were covered by several layers of PNrA/GO on the as-fabricated ammonia sensor (Fig. 1e). It has been clearly revealed in the high magnification Fig. If that graphene oxide nanosheets were decorated with tightly-arranged perpendicular PANI nanorod arrays.

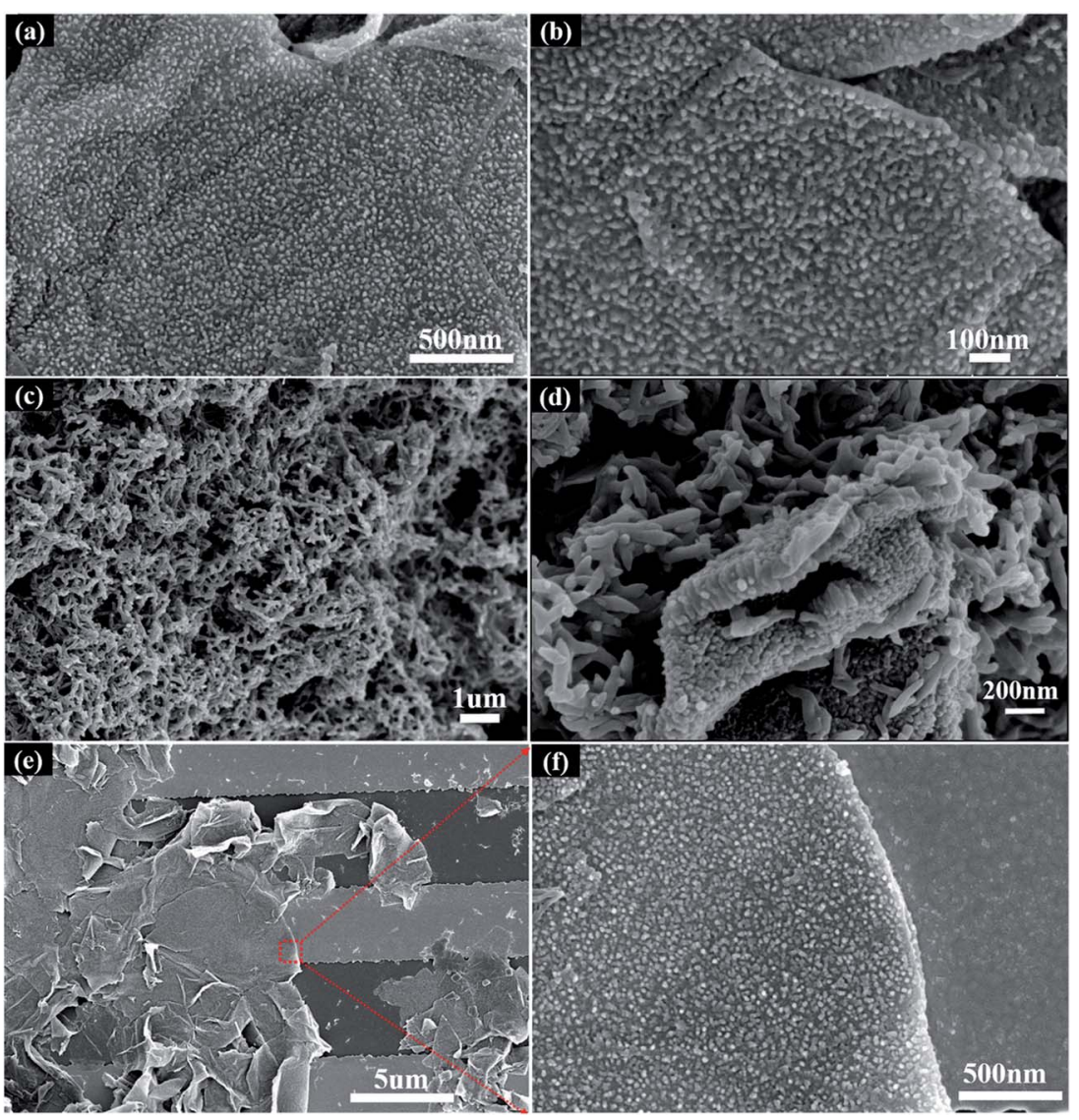

Fig. 1 (a) Low and (b) high magnification SEM images of vertically aligned PANI nanorod arrays grown on GO nanosheets (PNrA/GO). (c) SEM image of pure PANI nanofibers in interconnected network (PNF). (d) SEM image of PANI nanofibers synthesized with GO (PNF/GO). (e) Low and (f) high resolution SEM image of the ammonia sensor by drop-coating the as-prepared PNrA/GO onto interdigitated electrodes. 
On the basis of the above investigation, a detailed formation mechanism of PNrA/GO nanocomposite was further illustrated in Fig. S1. $\dagger$ In chemical polymerization of PANI, two possible nucleation mechanism, namely homogeneous nucleation and heterogeneous nucleation, coexist and compete with each other. Particularly, homogeneous nucleation usually dominates the process in high-concentration bulk solution, resulting in interconnected PANI nanofibers as shown in Fig. 1c and d, and yet the heterogeneous nucleation does in solution with foreign seeds or at the interface between GO and bulk solution. In Fig. S1b-d, $\uparrow$ the homogeneous polymerization was greatly suppressed in dilute aniline solution $(0.015 \mathrm{M}$ for Fig. S1b, $0.01 \mathrm{M}$ for Fig. S1c and $0.005 \mathrm{M}$ for Fig. S1d广) where a supersaturation state cannot be reached. Besides, the presence of abundant hydrophilic groups on GO nanosheets can attach to the anilinium ions via electrostatic attraction and serve as active nucleation sites for heterogeneous polymerization. The active sites would also minimize the interfacial energy barrier between the GO nanosheets and aniline solution, which was beneficial for the subsequent growth of PANI nanorod arrays on GO.
Besides, much longer and thicker PANI nanorod arrays appeared when the aniline concentration increased from $0.005 \mathrm{M}$ to $0.015 \mathrm{M}$, which would enhance the nanocomposite's sensing performances. However, the homogeneous growth also happened if the aniline concentration reached $0.02 \mathrm{M}$, and several segments of PANI nanowires and PANI nanorods agglomeration can be found on GO nanosheets in Fig. S1a, $\uparrow$ showing a strong tendency to form PANI thin film and PANI nanofibers.

To further characterize the heterogeneous growth of $\mathrm{PNrA} /$ GO nanocomposite, different samples were taken out from the reaction solution at reaction time of $1 \mathrm{~h}, 4 \mathrm{~h}, 8 \mathrm{~h}$ and $24 \mathrm{~h}$. Fig. 2a revealed the schematic illustration of the heterogeneous nucleation and growth process of $\mathrm{PNrA} / \mathrm{GO}$ at different reaction periods. And as shown in Fig. $2 b$, the attracted anilinium ions on GO nanosheets behaved as the nucleation sites to reduce the interfacial energy and the whole Gibbs free energy which facilitated further polymerization, and yet most areas of GO nanosheets remained smooth due to the slow reaction speed at low temperature. Fig. 2c showed the increased nucleation seeds and

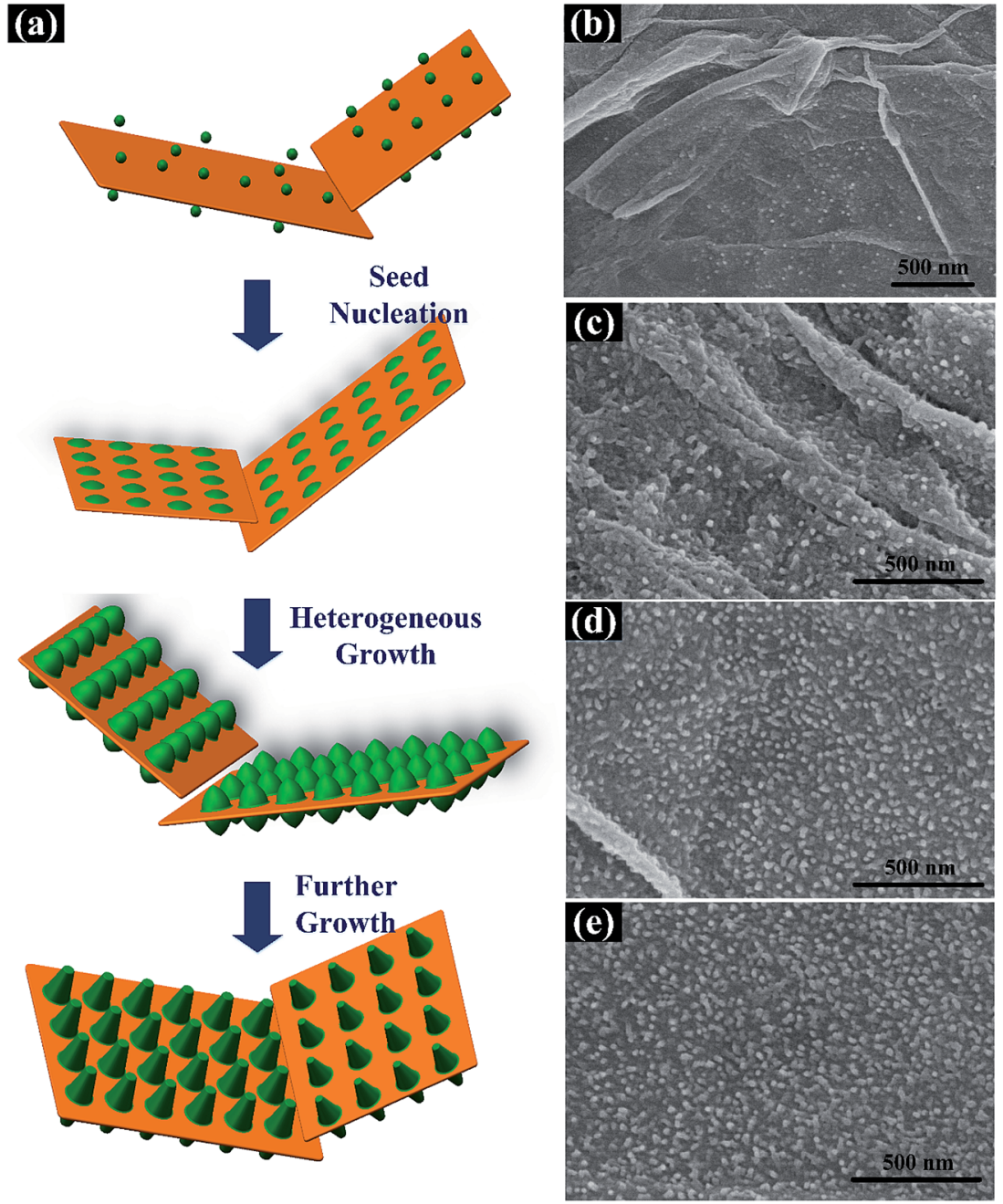

Fig. 2 (a) Schematic illustration of the heterogeneous nucleation and growth process of PNrA/GO at different reaction periods. And SEM images of PNrA/GO obtained at different reaction periods: (b) $1 \mathrm{~h}$; (c) $4 \mathrm{~h}$; (d) $8 \mathrm{~h}$; (e) $24 \mathrm{~h}$. 

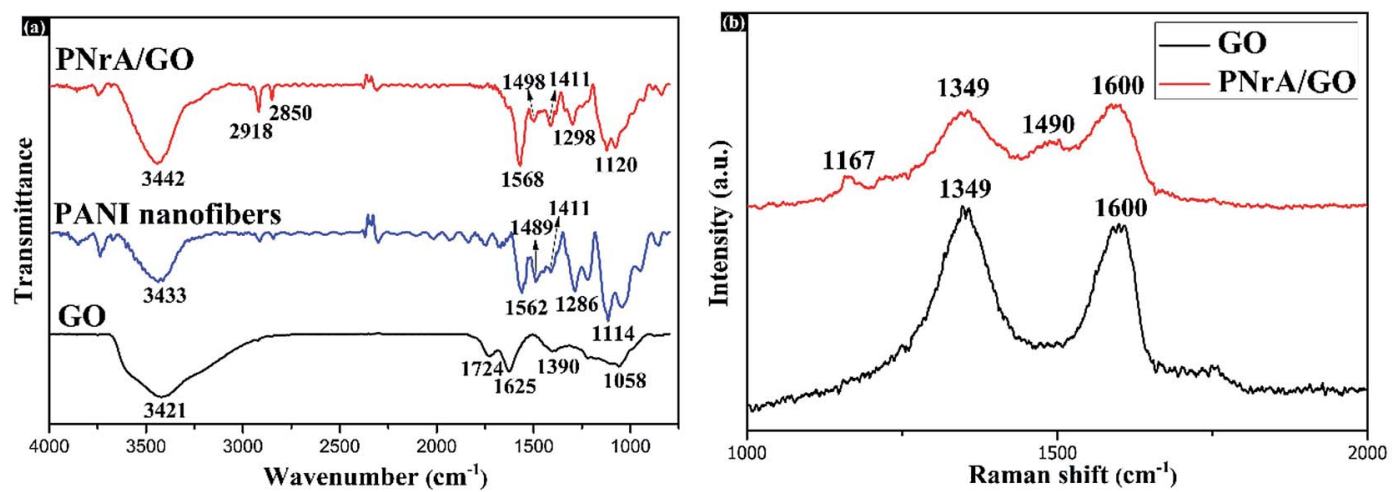

Fig. 3 (a) FTIR spectra of GO, PANI nanofibers and the as-prepared PNrA/GO (from bottom to top). (b) Raman spectra of GO and the asprepared PNrA/GO (from bottom to top).

the gradually-grown PANI nanoparticles on GO nanosheets. In the successive process as Fig. $2 \mathrm{~d}$ and e, the dispersed PANI nanoparticles developed into vertically aligned PANI nanorod arrays with uniform distribution on GO nanosheets, and the asprepared nanocomposite showed no appearance of particle-like PANI aggregates because of the dilute aniline. No PANI nanofibers was found due to the great homogeneous suppression effect in the dilute aniline solution. TEM images of the samples $(1 \mathrm{~h}, 4 \mathrm{~h}, 8 \mathrm{~h}$ and $24 \mathrm{~h}$ ) were also collected in Fig. S2 $\dagger$ to characterize the heterogeneous polymerization processes. It was demonstrated that PANI gradually developed from sparse nanoparticles (Fig. S2a †) into denser and longer rod-like nanoarrays (Fig. S2d $\dagger$ ).

The functional groups in the nanocomposite were further investigated by FTIR spectroscopy. In the FTIR spectrum of GO (Fig. 3a), the peaks at 3421, 1724, 1625, 1390 and $1058 \mathrm{~cm}^{-1}$ were attributed as the characteristic vibrations of the hydroxyl group, $\mathrm{C}=\mathrm{O}$ in $\mathrm{COOH}$, intercalated water, $\mathrm{O}=\mathrm{C}-\mathrm{O}$ from carboxylate, and $\mathrm{C}-\mathrm{O}$ in $\mathrm{C}-\mathrm{OH} / \mathrm{C}-\mathrm{O}-\mathrm{C}$ functional groups, respectively. ${ }^{19}$ In the case of pure PANI nanofibers, the peaks at
1562 and $1489 \mathrm{~cm}^{-1}$ were characterized to be the $\mathrm{C}=\mathrm{C}$ stretching vibrations in the quinoid and benzenoid units of PANI, respectively. And the peaks at 1286 and $1114 \mathrm{~cm}^{-1}$ were correspondingly attributed as the $\mathrm{C}-\mathrm{N}$ stretching vibrations in the quinoid and benzenoid units. Compared with GO, the appearance of characteristic peaks at 2918 and $2850 \mathrm{~cm}^{-1}$ in $\mathrm{PNrA} / \mathrm{GO}$ were demonstrated as the successful insertion of PANI oligomers into GO layers. Also, the distinct spectrum shown in pure PANI nanofibers were found in the $\mathrm{PNrA} / \mathrm{GO}$, further proving the combination of PANI nanostructures with GO nanosheets. It was noted that the peaks at 1724, 1390 and 1058 $\mathrm{cm}^{-1}$ disappeared in the prepared nanocomposite, which was attributed to the ion bonding behavior between anilinium cation $\left(\mathrm{C}_{6} \mathrm{H}_{5} \mathrm{NH}_{3}^{+}\right)$and $\mathrm{COO}^{-} / \mathrm{CO}^{-}$radicals of $\mathrm{GO}^{20,21}$

Structural investigation was sequentially carried out by Raman spectra, which is displayed in Fig. $3 b$. For all the samples, the distinctive peaks for D band $\left(1349 \mathrm{~cm}^{-1}\right)$ and G band $\left(1600 \mathrm{~cm}^{-1}\right)$ were observed for GO. Otherwise, the weaker D band and G band obtained for PNrA/GO could be due to the well coverage of PANI on GO nanosheets. ${ }^{22}$ In comparison, the
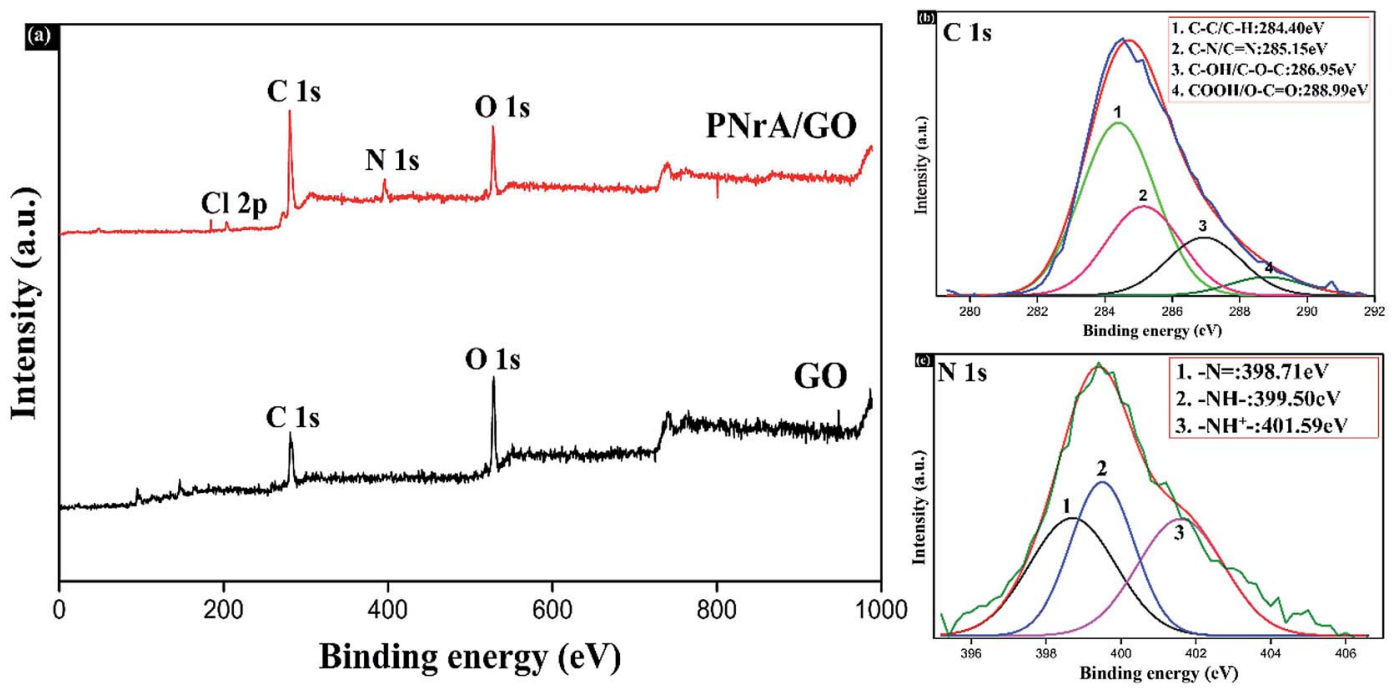

Fig. 4 (a) XPS spectra of GO and the as-prepared PNrA/GO (from bottom to top). High resolution XPS spectra and the fitting curves of (b) C 1s and (c) $\mathrm{N}$ 1s in the PNrA/GO. 
appearance of two characteristic peaks located at 1167 and 1490 $\mathrm{cm}^{-1}$ was in accordance with the $\mathrm{C}-\mathrm{N}$ stretching vibrations in the benzenoid units (1114 $\mathrm{cm}^{-1}$ ) and the $\mathrm{C}=\mathrm{C}$ stretching vibrations in the benzenoid units $\left(1489 \mathrm{~cm}^{-1}\right)$ of PANI in the FTIR spectrum as shown in Fig. 3a. And it demonstrated the existence of PANI in the as-synthesized nanocomposite. Furthermore, the $\mathrm{C}-\mathrm{N}$ stretching vibrations in the benzenoid units of PANI shifted to higher wavenumbers from $1114 \mathrm{~cm}^{-1}$ of pure PANI nanofibers to $1167 \mathrm{~cm}^{-1}$ in PNrA/GO nanocomposite, which indicated the $\pi-\pi$ conjugated interaction between PANI and GO nanosheets.

XPS characterization was broadly treated as an effective method to analyze the elemental composition and surrounding chemical states for functional nanomaterials. In the wide range spectra of Fig. 4a, both GO and the resulting PNrA/GO exhibited two strong peaks at $284.6 \mathrm{eV}$ and $529.1 \mathrm{eV}$ corresponding to the appearance of $\mathrm{C} 1 \mathrm{~s}$ and $\mathrm{O} 1 \mathrm{~s}$, respectively. Meanwhile, the existence of $\mathrm{N} 1 \mathrm{~s}$ and $\mathrm{Cl} 2 \mathrm{p}$ peaks was demonstrated in the PNrA/GO, indicating the presence of PANI, which was doped with $\mathrm{HClO}_{4}$. From the high resolution scanning spectrum for $\mathrm{C}$ 1s in Fig. 4b, it was observed that the binding energy at $284.40 \mathrm{eV}, 285.15 \mathrm{eV}, 286.95 \mathrm{eV}$ and $288.99 \mathrm{eV}$, derived from Gaussian fit, were reasonably attributed to the $\mathrm{C}-\mathrm{C}$ group, $\mathrm{C}-\mathrm{N}$ or $\mathrm{C}=\mathrm{N}$ groups in PANI frameworks, $\mathrm{C}-\mathrm{OH}$ or $\mathrm{C}-\mathrm{O}-\mathrm{C}$ groups in graphene oxide, and the $\mathrm{COOH}$ components, respectively. Also, the high resolution of $\mathrm{N} 1 \mathrm{~s}$ spectrum was deconvoluted into three Gaussian peaks (Fig. 4c) with the binding energy at $398.71 \mathrm{eV}, 399.50 \mathrm{eV}$ and $401.59 \mathrm{eV}$, which indicated the presence of benzenoid amine $(=\mathrm{N}-)$, quinoid amine $(-\mathrm{NH}-)$ and the nitrogen cationic radical $\left(-\mathrm{NH}^{+}-\right)$, respectively. In particular, the slight shifts of binding energy as observed in Fig. $4 \mathrm{~b}$ and c were largely due to $\pi-\pi$ conjugated stacking between the PANI frameworks and graphene oxide nanosheets. Additionally, the obtained XPS results revealed that the PANI nanorod arrays in the nanocomposite were in high-level doped state, which was in agreement with the good conductivity measured in the initial state.

\section{Evaluation of gas sensing properties}

As a toxic and corrosive chemicals widely existed in chemical industry, ammonia gas, especially in high concentrations, poses a great threat to human health, thus generating a strong urge for highly sensitive and selective ammonia gas sensor. Therefore, the as-prepared PNrA/GO here in a novel micro-structure was employed as sensitive material for ammonia sensor application.

The relative resistance changes of the as-fabricated gas sensors were then recorded when different gas concentrations were imposed on them. The typical gas response behaviors of the four ammonia gas sensors based on GO, PNF, PNF/GO and PNrA/GO nanocomposites towards $\mathrm{NH}_{3}$ vapor at various concentrations ranging from $50 \mathrm{ppm}$ to $400 \mathrm{ppm}$ were illustrated as Fig. 5a. It is widely known that PANI gas sensor will transform from the emeraldine salt form (conductive state) to its emeraldine base one (insulating one) on exposure to $\mathrm{NH}_{3}$ atmosphere, in which $\mathrm{NH}_{3}$ molecules display a strong deprotonation effect, leading the localization of polarons of conducting PANI and thus a dramatic decrease in conductance. $^{23}$ The deprotonation process is reversible, and the conductive state will restore with the decomposition of the PANI and $\mathrm{NH}_{3}$ molecules after the injection of fresh air. Conversely, a magnified view of GO-based gas sensor in Fig. S3† generated a slight decrease of resistance, showing n-type semiconducting properties in $\mathrm{NH}_{3}$ atmosphere, which can be attributed to the increased electron carrier concentration in GO nanosheets by the electron donating effect of $\mathrm{NH}_{3}$ molecules. In addition, the $\mathrm{PNF} / \mathrm{GO}$ nanocomposite reveal enhanced sensing response to the analyte compared to GO nanosheets or pure PANI nanofibers. Actually, the p-type PANI nanofibers can interact with the n-type semiconducting GO nanosheets through electrostatic attraction, hydrogen bonding and $\pi-\pi$ electron stacking and thus $\mathrm{p}-\mathrm{n}$ heterojunctions in the nanocomposite were formed for improved sensing performance. ${ }^{24-26}$ And it also demonstrated the highest response of PNrA/GO was obtained among those of all the nanocomposites. The enhanced synergistic effect between PANI and GO can be derived here from the larger number of PANI-GO heterojunctions, since the PANI nanorod arrays are synthesized in a uniform distribution on the GO nanosheets compared to PANI nanofibers/GO hybrid. ${ }^{12}$ And with regards to the decreased hole carrier concentration on exposure to the analyte, there would be an increase of depletion layer between PANI nanorod arrays and GO nano-sheets, which leads to reduced conducting pathways and enhanced sensing characteristics. ${ }^{27}$ Additionally, the vertically-grown PANI nanorod arrays will facilitate gas molecule diffusion process, which enable rapid and reversible adsorption and desorption kinetics with short response and recovery period. Hence, the asprepared PNrA/GO can be an ideal candidate for constructing a high-performance ammonia sensor with higher sensitivity and other required properties.

To illustrate the detailed sensing mechanism, the influence of vertically aligned PANI nanorod arrays on the ammonia sensing response was demonstrated in Fig. $5 \mathrm{~b}$ by comparing the sensing performances of different weight ratio of aniline to GO in as-synthesized nanocomposites. Fig. S4, $\uparrow$ a magnified view of PNrA/GO (1.3:1) in Fig. 5b, showed the similar negative response behaviors as that of pure GO nanosheets in Fig. S3, $\dagger$ which means sparse PANI nanoparticles have little positive effect and that GO dominate the sensing performance in the nanocomposite. In contrast, the PNrA/GO (5.2:1) in which the aniline concentration was raised to a high level, exhibited the similar sensing response just as that of PNF/GO in Fig. 5a. The trend here can be demonstrated to be in accordance with the morphological structure changes shown in Fig. S1.† Additionally, more interaction heterojunctions between PANI and GO were generated by the longer and thicker PANI nanorod arrays distributed on GO achieved in PNrA/GO (3.9:1) and thus the derived stronger synergistic effect lead to higher sensing response, compared to that of PNrA/GO (2.6:1). Therefore, the PNrA/GO (3.9:1) was chosen for other evaluations in the following sections, and a short name of PNrA/GO was taken for convenience. 

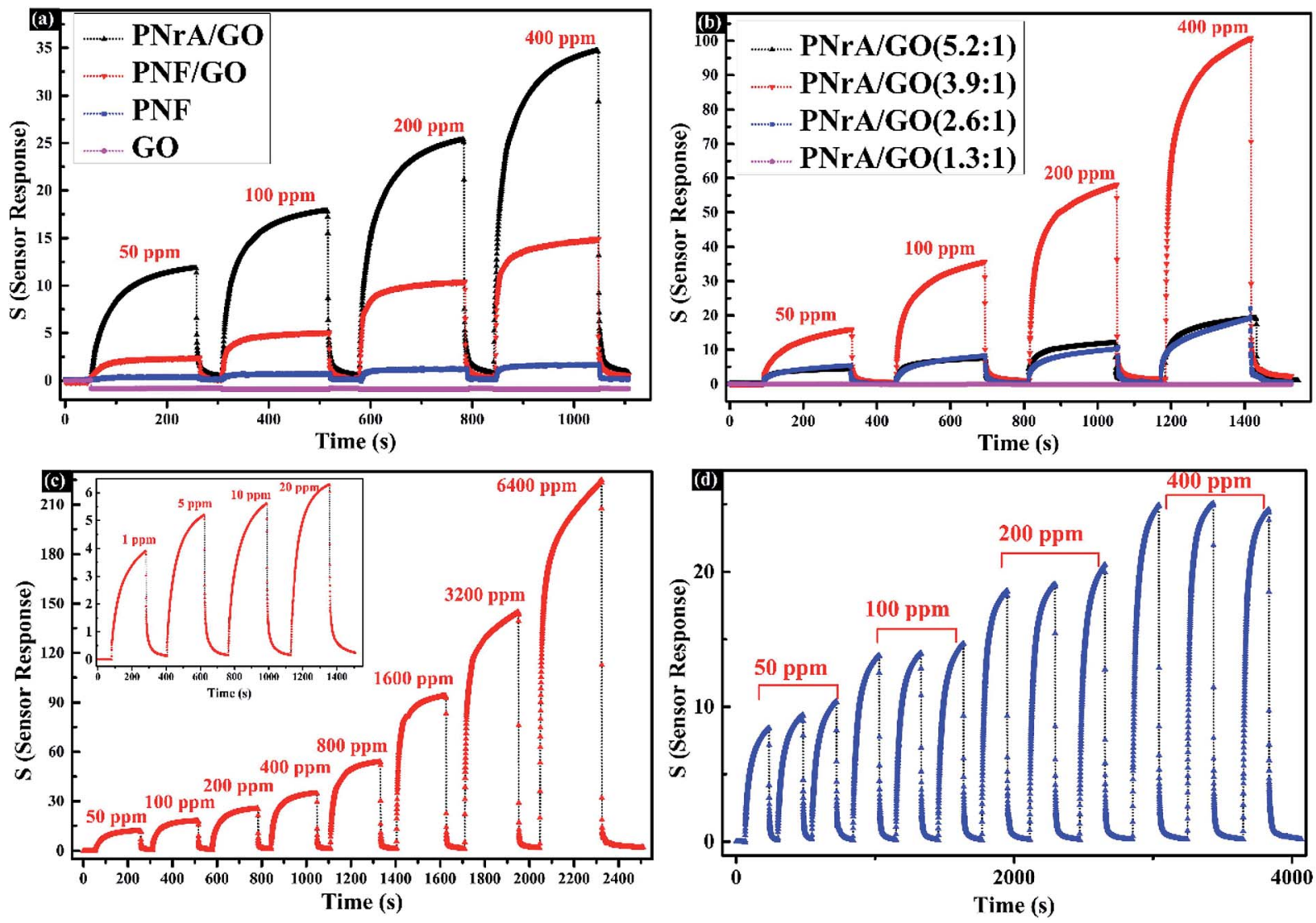

Fig. 5 (a) Gas response behaviors based on graphene oxide (GO), PANI nanofibers (PNF), PANI nanofibers/GO composite (PNF/GO) and the asprepared PNrA/GO towards 50,100, 200 and 400 ppm of $\mathrm{NH}_{3}$ in sequence at room temperature. (b) Gas sensitivity on exposure to $50,100,200$ and $400 \mathrm{ppm} \mathrm{NH}$ of PNrA/GO synthesized at different weight ratio of aniline to GO: $5.2: 1,3.9: 1,2.6: 1$ and $1.3: 1$. And the inset is a magnified view of PNrA/GO $(1.3: 1)$ to $\mathrm{NH}_{3}$. (c) Gas sensing performance of the PNrA/GO based ammonia sensor upon exposure to different concentration of $\mathrm{NH}_{3}$ ranging from $1 \mathrm{ppm}$ to $6400 \mathrm{ppm}$. Inset is the transient responses to 1, 5, 10 and $20 \mathrm{ppm}$ of $\mathrm{NH}_{3}$. (d) Gas sensing repeatability of the ammonia sensor based on PNrA/GO to 50, 100, 200 and 400 ppm of $\mathrm{NH}_{3}$.

In order to further investigate the detailed properties, the dynamic responses of $\mathrm{PNrA} / \mathrm{GO}$ to $\mathrm{NH}_{3}$ gas at various concentrations ranging from $1 \mathrm{ppm}$ to $6400 \mathrm{ppm}$ were evaluated in Fig. 5c. It showed the resistance of the as-prepared sensor experienced a sharp increase at $50 \mathrm{ppm}$ (sensor response of 14.63 to $\mathrm{NH}_{3}$ ) with the response time to be $61 \mathrm{~s}$ and recovery time as short as $10 \mathrm{~s}$ after sensor withdrawal. Dynamic range is always regarded as one of the most significant parameters for physical or chemical sensors. The fitting data of the response toward ammonia concentration ranging from $50 \mathrm{ppm}$ to 6400 ppm (Fig. S5†) shows the experimental data fits well with a two order polynomial $\left(R^{2}=0.997\right)$, which meant the fabricated ammonia sensor has a great potential for quantitative gas analysis in this concentration range..$^{28}$ In summary, the sensing performances of ammonia sensor based on $\mathrm{PNrA} / \mathrm{GO}$ were compared in Table 1 with other ammonia sensors previously reported. ${ }^{29,30}$ It revealed that the prepared ammonia sensor exhibited a relatively higher sensitivity and wider detection range.

As illustrated as Fig. 5d, the repeatability of the ammonia sensor was examined on exposure to the same gas concentration of 50,100, 200 and $400 \mathrm{ppm}$ in multiple cycles. And a satisfactory performance in repeatability was achieved from the consistency of the ascending and descending curves, indicating an unchanged adsorption and desorption kinetics at different times.

In Fig. 6a, the impact of the drop-coating amount of the $\mathrm{PNrA} / \mathrm{GO}$ on the sensing performances were carefully investigated in three ammonia sensors prepared by first dispersing the nanocomposite with deionized water in different volume ratio as $1: 1,1: 10$ and $1: 20$, and then drop-coating the same amount of diluted product onto the electrode. Here, the asfabricated ammonia sensors were named as PNrA/GO_1X, PNrA/GO_10X and PNrA/GO_20X, respectively. The highest gas sensitivity was obtained for PNrA/GO_20X, and yet the sensing behavior showed great nonlinearity to higher concentration of the ammonia vapor. With the decrease of the drop-coating amount, the nanocomposite stacked layers assembled on the electrode surface were gradually reduced to facilitate the interactions between the analyte with the inner composite layer, which made a great difference to the sensor response. In contrast, the PNrA/GO_1X exhibited a slower response and recovery behaviors, resulting in a slight upward baseline. And this was largely attributed to the slow diffusion of gas molecules 
Table 1 The comparison of the sensing performances between the as-fabricated ammonia sensor and other previously reported sensors

\begin{tabular}{|c|c|c|c|c|c|c|}
\hline $\begin{array}{l}\text { Sensitive } \\
\text { materials }\end{array}$ & $\begin{array}{l}\text { Working } \\
\text { temperature }\left({ }^{\circ} \mathrm{C}\right)\end{array}$ & Surface morphology & $\begin{array}{l}\text { Sensor response } \\
\text { to } 50 \mathrm{ppm} \mathrm{NH}_{3}\end{array}$ & $\begin{array}{l}\text { Detection range } \\
\text { (ppm) }\end{array}$ & $\begin{array}{l}\text { Response/recovery } \\
\text { time (s) }\end{array}$ & Ref. \\
\hline RGO/PANI & $\mathrm{RT}^{a}$ & Nanosheets/nanoparticles & 0.6 & $5-50$ & $500 / 240$ & 11 \\
\hline $\mathrm{RGO} / \mathrm{SnO}_{2} / \mathrm{PANI}$ & 25 & 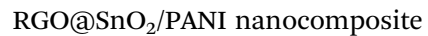 & 1.5 & $10-50$ & $600 / 1200$ & 17 \\
\hline $\mathrm{CeO}_{2} / \mathrm{PANI}$ & RT & Core-shell $\mathrm{CeO}_{2} @$ PANI & 6.5 & $2-400$ & $57.6 / 360$ & 27 \\
\hline $\mathrm{TiO}_{2} / \mathrm{PPy} / \mathrm{GN}$ & $\mathrm{RT}$ & Nanoparticle@PPy/graphene & 1.02 & $1-200$ & $36 / 16$ & 30 \\
\hline
\end{tabular}

${ }^{a}$ RT means room temperature here.

in the severe stacked layers of the nanocomposite. In summary, PNrA/GO_10X showed greater overall sensing performances when the sensitivity and detection range were balanced for optimizing the drop-coating amount of the sensing material to construct a high-performance gas sensor.

The selectivity of the prepared ammonia sensor was then demonstrated in Fig. $6 \mathrm{~b}$ and $\mathrm{S} 6 \dagger$ by exposing it to different gas species at $50 \mathrm{ppm}$, including $\mathrm{C}_{2} \mathrm{H}_{4}, \mathrm{CH}_{4}, \mathrm{C}_{4} \mathrm{H}_{8}, \mathrm{H}_{2} \mathrm{~S}, \mathrm{H}_{2}$ and $\mathrm{SO}_{2}$, and that the sensor response to $50 \mathrm{ppm} \mathrm{NH}_{3}$ was analyzed to be 14.63, much higher than that of other gas species $\left(0.38\right.$ of $\mathrm{C}_{2} \mathrm{H}_{4}$, 1.02 of $\mathrm{CH}_{4}, 1.18$ of $\mathrm{C}_{4} \mathrm{H}_{8}, 0.7$ of $\mathrm{H}_{2} \mathrm{~S}, 0.92$ of $\mathrm{H}_{2}$ and 0.15 of $\mathrm{SO}_{2}$ ). Generally, each gas species, due to their different molecule structures and the resultant interactions with the sensing nanocomposite, can produce different response mechanisms including protonation, deprotonation, reduction, swelling and conformational alignment. ${ }^{23}$ And the preferable selectivity
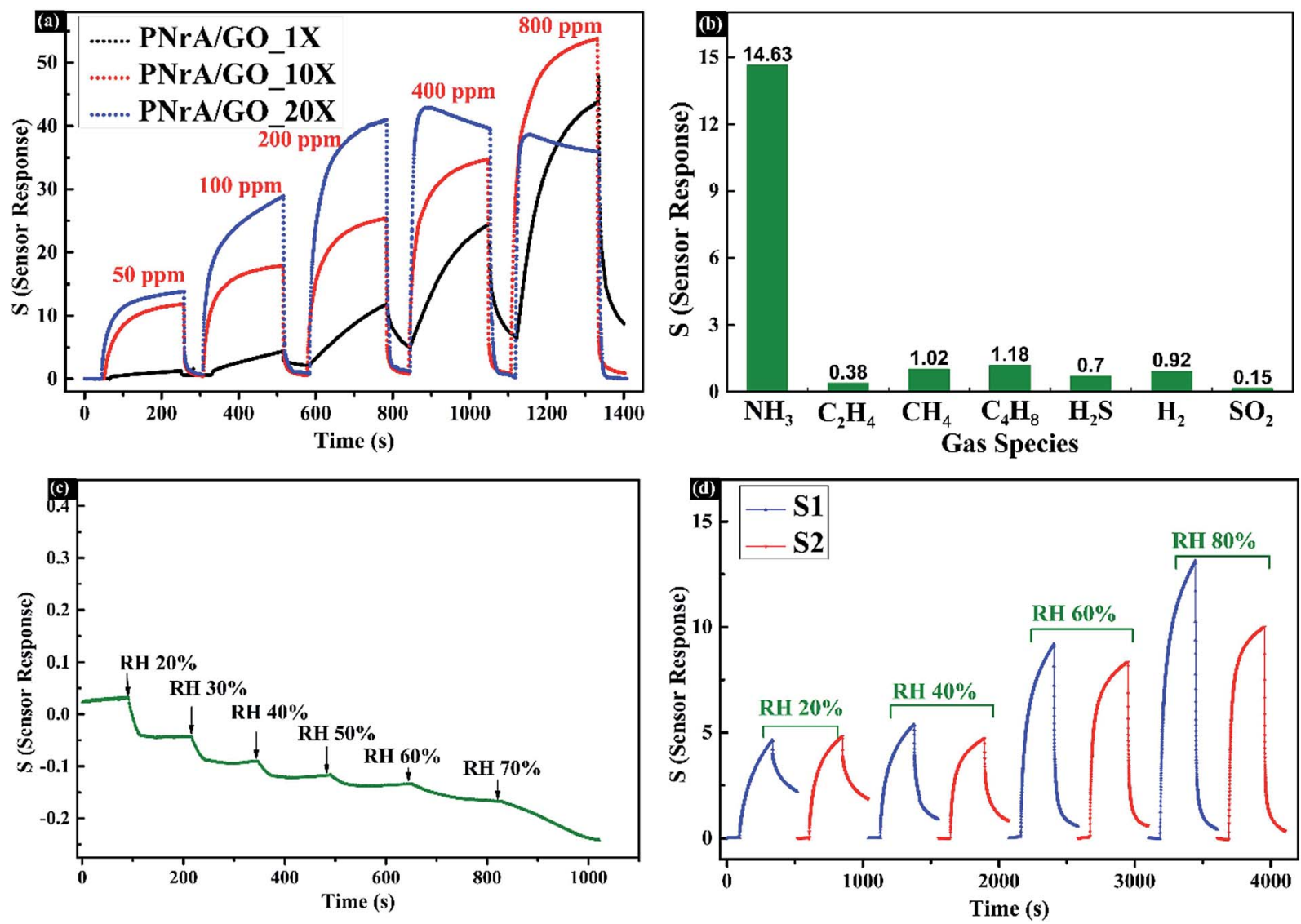

Fig. 6 (a) Gas sensing behaviors to 50,100, 200, 400 and 800 ppm of $\mathrm{NH}_{3}$ of PNrA/GO diluted by deionized water in different volume ratio as $1: 1,1: 10$ and $1: 20$ (named as PNrA/GO_1X,PNrA/GO_10X and PNrA/GO_20X, respectively). (b) Gas sensing selectivity of the ammonia sensor to various interfering gas species at concentration of $50 \mathrm{ppm}$, including $\mathrm{C}_{2} \mathrm{H}_{4}, \mathrm{CH}_{4}, \mathrm{C}_{4} \mathrm{H}_{8}, \mathrm{H}_{2} \mathrm{~S}, \mathrm{H}_{2}$ and $\mathrm{SO}_{2}$. (c) Sensor response behaviors of PNrA/GO based ammonia sensor under different relative humidity levels. (d) Gas sensing behaviors of PNrA/GO based ammonia sensor (sensor $\mathrm{S} 1$ and sensor S2 were fabricated in the same batch) to $50 \mathrm{ppm}$ of $\mathrm{NH}_{3}$ under different $\mathrm{RH}$ levels ranging from $20 \%$ to $80 \%$. 

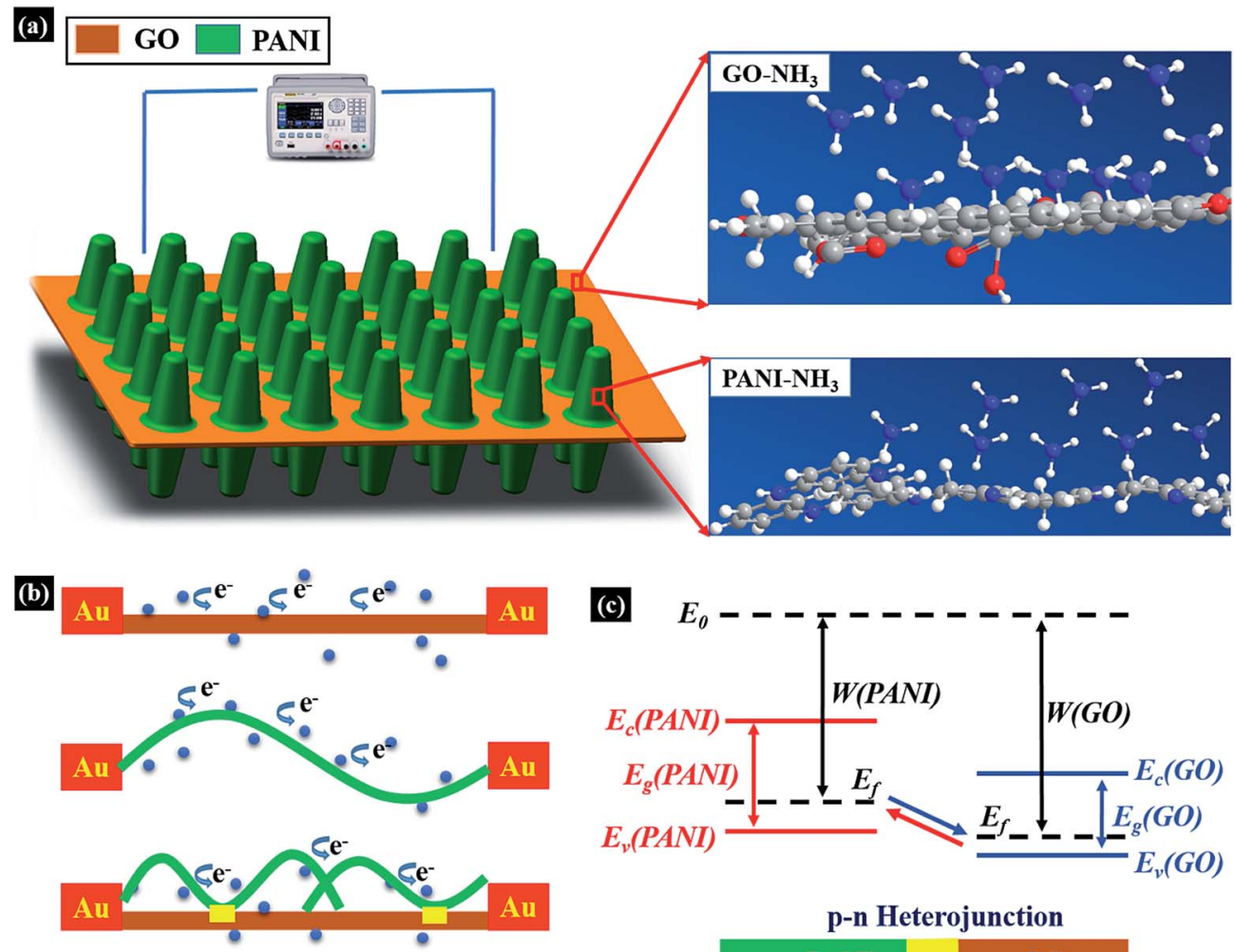

p-n Heterojunction
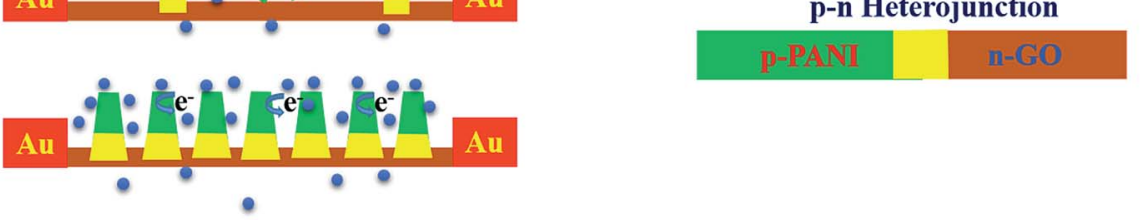

$\square \mathrm{n}$-GO $\square \mathrm{p}$-PANI $\square \mathrm{p}-\mathrm{n}$ heterojunction

$\square$ Ammonia $\square$ Au electrode

Fig. 7 (a) Schematic illustration of the interactions between $\mathrm{PNrA} / \mathrm{GO}$ and $\mathrm{NH}_{3}$ molecules at room temperature. (b) Formation and modulation mechanism of PANI/GO $n-p$ heterojunction in $\mathrm{NH}_{3}$ atmosphere. (c) Schematic energy band diagram of $p$-type PANI and $n$-type GO ( $E_{0}$ is vacuum energy level, $E_{\mathrm{f}}$ is Fermi energy level, $E_{\mathrm{c}}$ is the bottom of conduction band and $E_{\mathrm{v}}$ is the top of valence band).

performance of the proposed $\mathrm{PNrA} / \mathrm{GO}$ was largely attributed to the stronger deprotonation effect of $\mathrm{NH}_{3}$ to PANI in contrast to other interfering gases. Besides, the contradiction of desired selectivity and acceptable recovery time was compromised due to the simultaneous achievement of reversible deprotonation/ protonation reactions and specific chemical adsorption of $\mathrm{NH}_{3}$ molecules to PANI. Additionally, Fig. 6c revealed minor impacts on the sensor resistance changes caused by relative humidity at different levels from RH $20 \%$ to RH $70 \%$. However, different response patterns were clearly observed in Fig. 6d when gas sensor was exposed to $50 \mathrm{ppm} \mathrm{NH}_{3}$ at different $\mathrm{RH} \%$. In detail, both the two sensors fabricated in the same batch (abbreviated as S1 and S2) exhibited lower sensitivity in low $\mathrm{RH} \%$ as $20 \%$ and $40 \%$, and yet higher $\mathrm{RH} \%$ impose positive effects on the sensor response (Fig. S7 $\dagger$ ). Therefore, humidity compensation should be adopted when the sensor was used at different $\mathrm{RH} \%$. Moreover, the influence of working temperature on sensing performances was carefully investigated in Fig. S8. $\dagger$ It clearly revealed that the sensitivity of the as-fabricated ammonia sensor gradually decreased with the increase of the surrounding temperature from $10{ }^{\circ} \mathrm{C}$ to $70{ }^{\circ} \mathrm{C}$. It suggested that further efforts should be made to relieve the negative influences caused by humidity and temperature on the sensing characteristics.

\section{Sensing mechanism analysis}

Schematic illustration of the interactions between the nanocomposite and ammonia molecules at room temperature was revealed in Fig. 7a, where enhanced sensing response could be attributed to the effect of large number of $\mathrm{p}-\mathrm{n}$ hetero-junctions formed in the well-organized $\mathrm{PNrA} / \mathrm{GO}$ nano-composite. The interaction of $\mathrm{NH}_{3}$ with graphene oxides containing the hydroxyl and epoxy functional groups has been carefully investigated in first-principles calculations and experimental observations. ${ }^{24,31}$ It showed that great charge transferred from $\mathrm{NH}_{3}$ to $\mathrm{GO}$ when the adsorbed $\mathrm{NH}_{3}$ molecules on GO were decomposed into chemisorbed $\mathrm{NH}_{2}$ species as a result of the $\mathrm{H}$ atom abstraction, and that the introduction of nitrogen functionalities converted the insulating GO to n-type GO and narrowed the band gap. ${ }^{26,32}$ The n-type GO can also be observed in Fig. $\mathrm{S} 3 \uparrow$ where the resistance obviously decreased upon 
exposure to $\mathrm{NH}_{3}$. In contrast, the hole concentration in positively charged PANI backbone was reduced by electron-donating $\mathrm{NH}_{3}$ molecules due to the dedoping reaction. And in this case, the p-type PANI transform from the emeraldine salt form (conductive state) to its emeraldine base one (insulating one). Therefore, $\mathrm{p}-\mathrm{n}$ heterojunctions were formed in the PANI/GO nanocomposite when exposed to the $\mathrm{NH}_{3}$ atmosphere.

To further understand synergistic effect of the heterojunction, the modulation mechanism and energy band diagram of PANI/GO junction was illustrated in Fig. 7b and c. The band gaps of p-type PANI and n-type GO were $2.8 \mathrm{eV}$ (ref. 27) and $2.2 \mathrm{eV},{ }^{26}$ respectively, and a $\mathrm{p}-\mathrm{n}$ heterojunction was formed at the interface of PANI and GO, due to the different Fermi energy level. In the case of PANI nanofibers/GO composite, carrier transportation could find pathways in inter-chain or intra-chain PANI backbones, and heterojunctions formed between PANI nanofibers and GO may slightly enhance the sensing performance compared to bare PANI nanofibers. When the PANI nanorod arrays were uniformly distributed on GO nanosheets, enhanced synergistic effect was derived from the larger number of $\mathrm{p}-\mathrm{n}$ heterojunctions and the depletion region in the nano-composite may be increased by $\mathrm{NH}_{3}$ electrondonating and energy band modulation effect, which greatly blocked the carrier conducting pathways and attributed to the excellent sensing responses.

\section{Conclusions}

Vertically aligned PANI nanorod arrays could be facilely synthesized via an in situ dilute polymerization method with aniline molecules as precursors and GO nanosheets as soft support. The homogenous growth of PANI was greatly suppressed in ultralow concentrations, which made uniformly distributed PANI nanorod arrays grow on GO in a heterogeneous way. An ammonia gas sensor based on the resulting composite was then successfully fabricated. Compared with other nanocomposites based ammonia sensors, it exhibited excellent sensitivity, wider dynamic range to $\mathrm{NH}_{3}$ of $1 \mathrm{ppm}$ to $6400 \mathrm{ppm}$, short response and recovery time of several tens of seconds, as well as favorable selectivity when exposed to other disturbing gases. Here, the large number of $\mathrm{p}-\mathrm{n}$ heterojunctions between PANI and GO in the strategically designed microstructure enhanced the synergistic effect, and contributed to these remarkable characteristics of the sensor. Besides, the most suitable drop-coating amount of the nanocomposite was demonstrated to achieve the desired sensing performances. In general, effective methods for new microstructure design and sensor fabrication optimization will pave a new way for further improvements to high-performance gas sensor.

\section{Acknowledgements}

This work was financially supported by the National Natural Science Foundation of China (No. 60875078, 61501400), China Postdoctoral Science Foundation (2015M571879), Special Foundation of China Postdoctoral Science (2016T90541) and Cultural Relics Protection Science and Technology Project of Zhejiang Bureau of Cultural Relics (2015015).

\section{Notes and references}

1 F. Schedin, A. K. Geim, S. V. Morozov, E. W. Hill, P. Blake, M. I. Katsnelson and K. S. Novoselov, Nat. Mater., 2007, 6, 652-655.

2 D. Ghosh, S. Giri, M. Mandal and C. K. Das, $R S C A d v$., 2014, 4, 26094-26101.

3 S. Giri, D. Ghosh and C. K. Das, Adv. Funct. Mater., 2014, 24, 1312-1324.

4 F. Schwierz, Nat. Nanotechnol., 2010, 5, 487-496.

5 H. Choi, J. S. Choi, J.-S. Kim, J.-H. Choe, K. H. Chung, J.-W. Shin, J. T. Kim, D.-H. Youn, K.-C. Kim, J.-I. Lee, S.-Y. Choi, P. Kim, C.-G. Choi and Y.-J. Yu, Small, 2014, 10, 3685-3691.

6 F. Yavari and N. Koratkar, J. Phys. Chem. Lett., 2012, 3, 17461753.

7 A. Gutes, B. Hsia, A. Sussman, W. Mickelson, A. Zettl, C. Carraro and R. Maboudian, Nanoscale, 2012, 4, 438-440.

8 S. Liu, Z. Wang, Y. Zhang, Z. Dong and T. Zhang, RSC Adv., 2015, 5, 91760-91765.

9 W. J. Yuan, L. Huang, Q. Q. Zhou and G. Q. Shi, ACS Appl. Mater. Interfaces, 2014, 6, 17003-17008.

10 S. Bai, Y. Zhao, J. Sun, Y. Tian, R. Luo, D. Li and A. Chen, Chem. Commun., 2015, 51, 7524-7527.

11 X. Huang, N. Hu, R. Gao, Y. Yu, Y. Wang, Z. Yang, E. S.-W. Kong, H. Wei and Y. Zhang, J. Mater. Chem., 2012, 22, 22488-22495.

12 Z. Wu, X. Chen, S. Zhu, Z. Zhou, Y. Yao, W. Quan and B. Liu, Sens. Actuators, B, 2013, 178, 485-493.

13 L. Al-Mashat, K. Shin, K. Kalantar-zadeh, J. D. Plessis, S. H. Han, R. W. Kojima, R. B. Kaner, D. Li, X. Gou, S. J. Ippolito and W. Wlodarski, J. Phys. Chem. C, 2010, 114, 16168-16173.

14 X. Feng, N. Chen, J. Zhou, Y. Li, Z. Huang, L. Zhang, Y. Ma, L. Wang and X. Yan, New J. Chem., 2015, 39, 2261-2268.

15 D. Shao, G. Hou, J. Li, T. Wen, X. Ren and X. Wang, Chem. Eng. J., 2014, 255, 604-612.

16 S. Zhang, M. Zeng, W. Xu, J. Li, J. Li, J. Xu and X. Wang, Dalton Trans., 2013, 42, 7854-7858.

17 Z. Ye, Y. Jiang, H. Tai, N. Guo, G. Xie and Z. Yuan, J. Mater. Sci.: Mater. Electron., 2015, 26, 833-841.

18 X. Huang, N. Hu, L. Zhang, L. Wei, H. Wei and Y. Zhang, Synth. Met., 2013, 185, 25-30.

19 S. Park, K.-S. Lee, G. Bozoklu, W. Cai, S. T. Nguyen and R. S. Ruoff, ACS Nano, 2008, 2, 572-578.

20 Y. Matsuo, T. Niwa and Y. Sugie, Carbon, 1999, 37, 897-901. 21 S.-J. Lin, H.-J. Sun, T.-J. Peng and L.-H. Jiang, High Perform. Polym., 2014, 26, 790-797.

22 Y. Luo, D. Kong, Y. Jia, J. Luo, Y. Lu, D. Zhang, K. Qiu, C. M. Li and T. Yu, RSC Adv., 2013, 3, 5851-5859.

23 S. Virji, J. Huang, R. B. Kaner and B. H. Weiller, Nano Lett., 2004, 4, 491-496.

24 S. Tang and Z. Cao, J. Phys. Chem. C, 2012, 116, 8778-8791. 25 C. Petit, M. Seredych and T. J. Bandosz, J. Mater. Chem., 2009, 19, 9176-9185. 
26 T.-F. Yeh, S.-J. Chen, C.-S. Yeh and H. Teng, J. Phys. Chem. C, 2013, 117, 6516-6524.

27 L. Wang, H. Huang, S. Xiao, D. Cai, Y. Liu, B. Liu, D. Wang, C. Wang, H. Li, Y. Wang, Q. Li and T. Wang, ACS Appl. Mater. Interfaces, 2014, 6, 14131-14140.

28 X. Wang, S. Zhang, M. Shao, J. Huang, X. Deng, P. Hou and X. Xu, Sens. Actuators, B, 2017, 251, 27-33.

29 P. Wan, X. Wen, C. Sun, B. K. Chandran, H. Zhang, X. Sun and X. Chen, Small, 2015, 11, 5409-5415.
30 C. Xiang, D. Jiang, Y. Zou, H. Chu, S. Qiu, H. Zhang, F. Xu, L. Sun and L. Zheng, Ceram. Int., 2015, 41, 6432-6438.

31 E. C. Mattson, K. Pande, M. Unger, S. Cui, G. Lu, M. Gajdardziska-Josifovska, M. Weinert, J. Chen and C. J. Hirschmugl, J. Phys. Chem. C, 2013, 117, 10698-10707. 32 F. Li, X. Jiang, J. Zhao and S. Zhang, Nano Energy, 2015, 16, 488-515. 